\title{
Analysis about the traffic violation that avoid the coil detection by the automatic recording system of running red light
}

\author{
LuZhenyi $^{1, a}$, Guoyong ${ }^{2, b}$, WuChangcheng ${ }^{1, c}$ \\ ${ }^{1}$ Traffic Management Research Institute of Public Security, Wuxi, China, 214151. \\ ${ }^{2}$ Wuxi Huatong intelligent traffic technique development limited company, Wuxi, China, 214151 \\ aluzhenyi_1976@163.com, b seagreen@126.com, dingsouqing@163.com
}

Keywords: running red lights, coil, traffic violations, traffic safety

\begin{abstract}
With the automatic recording system of running red light widely used in traffic management, traffic violation of running red light has much suppressed. Since the automation recording system use the coil to detect the vehicles, parts of the vehicle drivers arouse some new traffic violations in order to avoid the detection by the coil, making the automatic recording system of running red light cannot monitor their traffic violation. This paper first describes how the automatic recording system of running red light use the coil to detect the vehicle when they passing through the road intersection. Then, it analyzes the danger about the traffic violation that avoids the detection by the coil in the automatic recording system. At last, it gives some advice to improve the monitoring function about the automatic recording system, improving the traffic safety of the vehicle and the pedestrian when they crossing the road intersection.
\end{abstract}

\section{Introduction}

With the rapid development of social economy and sharp increase of vehicle populations, the traffic facilities available cannot meet the increasing traffic demand, which has resulted in the regular traffic jam and traffic accidents and brought great economic losses. For the traffic accidents occurred on the road intersection, most of them are traffic signal violation and traffic direction violation, caused by the vehicles passing through the road without following the traffic signals and the desired traveling direction. Therefore, it is necessary to suppress these traffic signal violations, aiming to improve the road traffic safety on the vehicles and pedestrian.

Traffic signal violation refers to the behaviors that the vehicles violate the traffic rules under the red light phase on the road intersection or the road rad section, crossing the stop line and continuing traveling. According to the road traffic safety law and the road traffic safety provisions in china, if the vehicles cross the road without following the traffic signals when entering the signalized road intersection, the vehicle drivers will be judged as the traffic signal violation, with the main three situations as following:(1)Vehicles turn right when the right-turn red lamp is lighted; (2) Vehicles go through when the straight-through red lamp is lighted; (3) Vehicles turn left when the left-turn red lamp is lighted.

Traffic direction violation refers to the behaviors that the vehicles enter the approach lane without following the desired traveling direction. According to the road traffic safety law and the road traffic safety provisions, if the vehicles enter the approach lane without following the traveling direction when entering the signalized road intersection, the vehicle drivers will be judged as the traffic direction violation, with the main three situations as following: (1) Vehicles go through or turn right when entering the right-turn lane; (2) Vehicles turn left or turn right when entering the straight-through lane; (3) Vehicles go through or turn right when entering the left-turn lane.

At present, the automatic recording system of running red light has been widely used to suppress the traffic violation at the road section, using the coil to detect the vehicles when they passing through the road intersection. The traffic safety on the vehicles and pedestrian has been widely improved when they crossed the road intersection or road section. However, at present, parts of the vehicle drivers arouse some new traffic violations in order to avoid the coil detection by the automatic recording system of running red light. In this paper, we firstly analysis these traffic 
violation, and then give some advice to improve the monitoring function about the automatic recording system of running red light. Experiment results show that the proposed method greatly suppress these traffic violation and improve the traffic safety of the vehicle and the pedestrian at the road intersection.

\section{Brief introduction about the automatic recording system of running red light}

The automatic recording system of running red light is mainly consisted of the traffic signal detection unit, the vehicle detection unit, and the image collection unit. When the automatic recording system of the running red light is in operation, the traffic signal is switched in the signal detection unit to judge whether the lane is forbidden or pass. At the same time, the vehicle detection unit detects whether the vehicle pass the coil. When the signal detection unit detects that the lane is forbidden and the vehicle detection unit detects the vehicle passes the coil, the image collection unit will take the image of traffic violation vehicle.

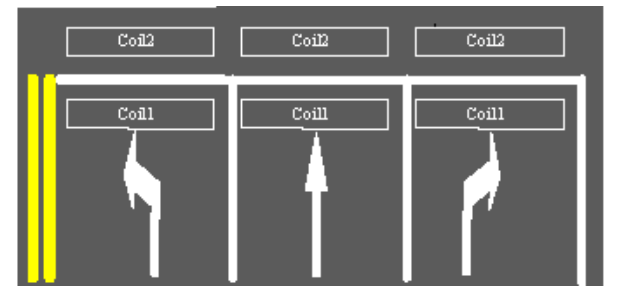

Figure.1 position about the coil bedded under the lane

The coil is the cable, surrounded several circles and bedded under the lane. When the vehicle crosses the lane, the inductance of the coil will be changed so as to judge whether the vehicle passed through the lane. Fig. 1 shows the positions about the coil bedded under the lane. Let us take the left-turn lane as the example to show how the coil detects the traffic violation vehicles. When the left-turn signal is green, even if the vehicle crosses the coil 1 and coil 2, the automatic recording system of running red light will judge the vehicle as normal travel. When the left turning signal is red, if the coil 1 detects the vehicle and the vehicle does not cross the stop line, the image collection unit will take the photo of the vehicle. When the vehicle continues the travel and crosses the stop line, the coil 2 detects the vehicle and the image collection unit will take the photos of the vehicle.

\section{Analysis about the traffic violation that avoid the coil detection by the automatic recording system of the running red light}

Although the automatic recording system of running the red light has been widely used to suppress the traffic violation at the road intersection, parts of the vehicle drivers arouse some new traffic violations in order to avoid the coil detection, bringing the high harm to the safety of the vehicles and the pedestrian.

\section{Traffic violation on the left-turn lane}

When the left-turn signal is green and straight-through and right-turn signals are red, although the vehicle can be detected by the coil1 and coil2 on the left-turn lane, the automatic recording system of running red light judges the vehicle as the normal travel. However, if the vehicles turn into straight-through or right-turn lane, it will conflict with other vehicles, leading to the potential traffic safety hazard. Two cases can be exampled as following, with the traffic signal assumed that the left-turn direction of vehicles on the north and south entry are set as the green phase, together, the right-turn direction of vehicles on the east and west entry are set as the green phase, while the other directions are set as red phase.

Straight-through change: As shown in Fig.2a, when the left-turn vehicle in the south entry changes into straight-through lane for the travel on north exit after passing through the coil1 and coil2, the automatic recording system of running the red light confirms the vehicle as normal travel. However, it will lead the potential traffic hazards to the left-turn vehicles at the north exit, the left-turn non-motor vehicles at the south entry and left-turn non-motor vehicles at the north entry.

Right-turn change: As shown in Fig.2b, when the left-turn vehicle in the south entry changes 
into right-turn lane for the travel on east exit after passing through the coil1 and coil2, the automatic recording system of running the red light confirms the vehicle as normal travel. However, it will lead the potential traffic hazards to left-turn non-motor vehicles at the south entry and the left-turn non-motor vehicles at the north entry.

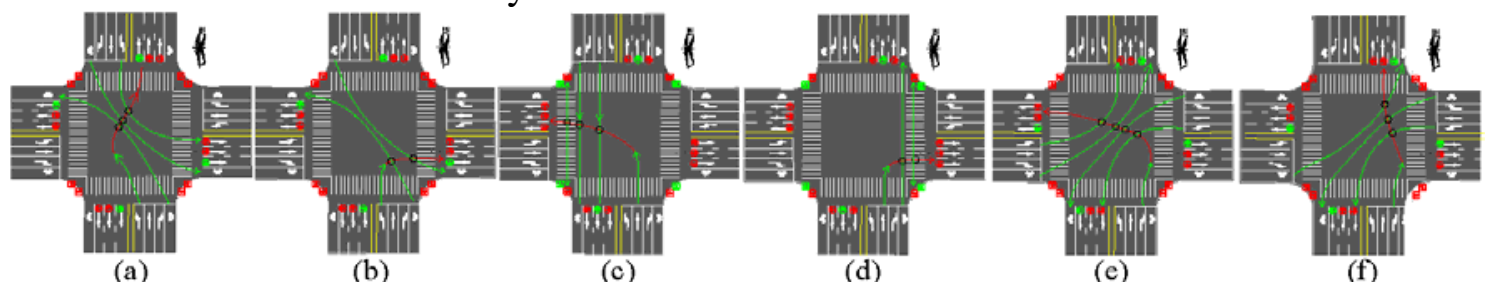

Figure.2 Traffic violation avoid the coil detection by the automatic recording system of the running red light : $(a, b)$ left-turn lane: $(b, c)$ straight-through lane; $(e, f)$ right-turn lane

\section{Traffic violation on the straight-through lane}

When the straight-through signal is green and left-turn and right-turn signals are red, although the vehicle can be detected by the coil1 and coil2 on the straight-through lane, the automatic recording system of running red light judges the vehicle as the normal travel. However, if the vehicles turn into left-through or right-turn lane, it will conflict with vehicles, leading to the potential traffic safety hazard. Two cases can be exampled as following, with the traffic signal assumed that the straight-through direction of vehicles on the north and south entry are set as the green phase, together, the direction of pedestrian on the north and south are set as the green phase, while the other directions are set as red phase.

Left-turn change: As shown in Fig.2c, when the straight-through vehicle in the south entry changes into left-turn lane for the travel on west exit after passing through the coil1 and coil2, the automatic recording system of running the red light confirms the vehicle as normal travel. However, it will lead the potential traffic hazards to the straight-through vehicles at the north entry, the straight-through non-motor vehicles at the south entry and the pedestrian .

Right-turn change: As shown in Fig.2d, when the left-turn vehicle in the south entry changes into right-turn lane for the travel on east exit after passing through the coil1 and coil2, the automatic recording system of running the red light confirms the vehicle as normal travel. However, it will lead the potential traffic hazards to straight-through non-motor vehicles at the south entry and the pedestrian.

\section{Traffic violation on the right-turn lane}

When the right-turn signal is green and left-turn and straight-through signals are red, although the vehicle can be detected by the coil1 and coil2 on the right-turn lane, the automatic recording system of running red light judges the vehicle as the normal travel. However, if the vehicles turn into straight-through or left-turn lane, it will conflict with other vehicles, leading to the potential traffic safety hazard. Two cases can be exampled as following, with the traffic signal assumed that the right-turn direction of vehicles on the north and south entry are set as the green phase, together, the left-turn direction of vehicles on the east and west are set as green phase, while the other directions are set as red phase.

Left-turn change: As shown in Fig.2e, when the left-turn vehicle in the south entry changes into right-turn lane for the travel on east exit after passing through the coil1 and coil2, the automatic recording system of running the red light confirms the vehicle as normal travel. However, it will lead the potential traffic hazards to vehicles at the east and west, and left-turn non-motor vehicles at the east and west either.

Straight-through change: As shown in Fig.2f, when the right-turn vehicle in the south entry changes into left-turn lane for the travel on west exit after passing through the coil1 and coil2, the automatic recording system of running the red light confirms the vehicle as normal travel. However, it will lead the potential traffic hazards to the left-turn vehicles and the on-motor vehicles at the east entry, the left-turn non-motor vehicles at the west entry. 


\section{Method about monitoring the traffic violation that avoid the coil detection by the automatic recording system of the running red light}

From the above discussions, traffic violation that avoids the coil detection by the automatic recording system of running the red light brings high hazard to the safety of the vehicles and pedestrian. Aiming to the faults of coil detection about the automatic recording system of running red light, we suggest the visual detection method here collect the traveling tracks of the vehicles when they passing through the road section. If the vehicles crossed the road intersection without following the approach lane, they will be judged as traffic violation. Method about using the visual detection on these traffic violations can be referenced in Ref.[3], Ref.[4] and Ref.[5].

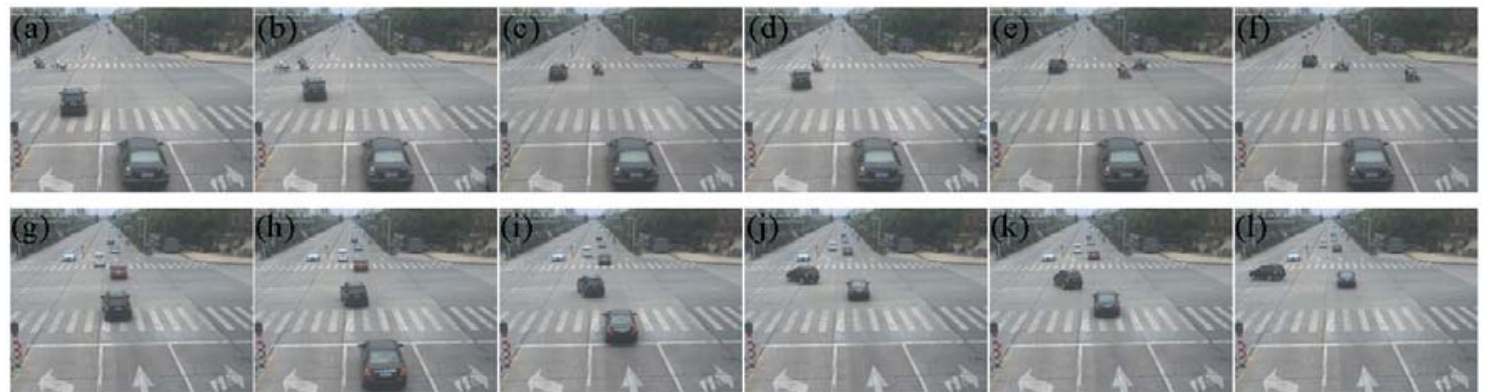

Figure.3 track about traffic violation that avoid the coil detection: (a)-(f) left-turn lane experiment; (g)-(l) straight-through lane experiment

Let us take the typical road intersection as the experimental analysis. The experiment is done on the road intersection of Zhouxin Road-Wanshun Road, Wuxi City, Jiangsu Province on June 32012. Because traffic signal of this road intersection is combined of left-turn and disc, we take the left-turn traffic signal and the straight-through signal as the experiment analysis.

Traffic violation on Left-turn lane experiment: Fig.3a to Fig.3c shows that the vehicles travel normally on turn-left lane. Fig.3d to Fig.3f shows that the vehicles change from left-turn lane into straight-through lane.

Traffic violation on straight-through lane experiment: Fig.3g to Fig.3i shows that the vehicles travel normally on straight-through lane. Fig.3j to Fig.3l shows that the vehicles change from straight-through into left-turn lane.

For the above experimental analysis, the visual detection method successfully monitors the traffic violation that avoids the coil detection of the automatic recording system of running red light, powerfully manage these traffic violations and highly improve the safety of the vehicles and pedestrian on the road intersection.

\section{Summary}

Considering the working principle of the automatic recording system of running red light, this paper firstly analyses how it use the coil to detect the traffic violation vehicles. Then, it describes other traffic violations that avoid the detection by the coil. Finally, it proposes some visual advice to avoid the defaults of the coil detection in the automatic recording system of running red light. Experimental results show that the proposed method successfully monitor these traffic violation and highly improve the traffic safety of the vehicles and pedestrian on the road intersection.

\section{Reference}

[1] Public safety industry standard. Specifications for the construction of public security traffic command system. GA/T455-2010

[2] Public safety industry standard. General specifications of automatic detecting and recording system of vehicles for violation of traffic signal. GA/T 496-2009.

[3] Magee D. Tracking multiple vehicle using foreground, background and motion models. Image and Vision Computing, 2004,22(2):143-155

[4] Deguchi Koichiro, Kawanaka Oki, Okatani Takayuki. Object tracking by the mean shift of 
regional color distribution com2bined with the particle-filter algorithm. United Kingdom: Institute of Electrical and Electronics Engineers Inc, 2004:506-509.

[5] Maggio Emilio, Cavallaro Andrea. Hybrid particle filter and mean shift tracker with adaptive transition model. United States: Institute of Electrical and Electronics Engineers Inc, 2005:221-224.

[6] Public safety industry standard. Technology specifications for video forensics devices of road traffic violation. GA/T995-2010 